\title{
EULAR/European news
}

\section{Citrullination and autoimmune disease: 8th Bertine Koperberg meeting}

$\mathrm{C}$ itrullination, the process in which citrulline-containing proteins are formed, seems to have a role in several autoimmune diseases. ${ }^{12}$ Although citrulline is a common metabolite present throughout the human body, it is a non-standard amino acid, which means that it cannot be incorporated into proteins during protein synthesis. Citrulline-containing proteins can only be generated through post-translational modification of arginine residues, a reaction that is catalysed by peptidylarginine deiminase (PAD) enzymes. $^{3}$

At the 8th Bertine Koperberg meeting in Nijmegen, The Netherlands, held in March 2005, the way in which citrullination might be involved in the autoimmune diseases rheumatoid arthritis (RA) and multiple sclerosis (MS) was discussed.

\section{PAD ENZYMES, CITRULLINATION, AND ANTIBODIES TO CITRULLINATED PROTEINS}

Ger Pruijn (Nijmegen, The Netherlands) gave an excellent overview of the enzymes responsible for citrullination. Five, highly conserved isotypes of PAD enzymes exist in all mammalian species (PADI-PAD4 and PAD6). The main difference between the isotypes is their tissue-specific expression. For RA and MS, PAD2 and PAD4 are the most interesting enzymes as both of them are expressed in the synovium and central nervous system (CNS). All PAD enzymes require high calcium levels for activity (about $\left.10^{-5} \mathrm{~mol} / \mathrm{l}\right)$. Because the intracellular calcium concentration in healthy cells is much lower $\left(\sim 10^{-7} \mathrm{~mol} / \mathrm{l}\right)$, the enzymes are only activated during events that lead to high calcium levels, like cell death, or late in the differentiation of skin cells (PAD1 and PAD3), as was explained by Michel Simon (Toulouse, France). Citrullination thus is believed to have an important role in several physiological processes, including the normal development of the myelin sheath and epidermis.

The effect of citrullination on protein function was also discussed. Whereas arginine is a positively charged amino acid, citrulline is uncharged, and citrullination of proteins thus leads to a loss of net positive charge. As a result, the target proteins adopt an altered, generally more open structure. This change in structure may severely hamper the cellular function of these proteins. Interestingly, all known natural substrates of PAD enzymes are proteins that have an important structural function (for example, intermediate filament proteins, myelin basic protein (MBP), histones, keratins, fibrin $\operatorname{clots}^{12}$ ).

The question whether the presence of citrullinated antigens such as fibrin and vimentin is specific for RA was extensively discussed. Mireille Sebbag (Toulouse, France) and Erik Vossenaar (Nijmegen, The Netherlands) presented data indicating that citrullinated proteins are generated during many different types of inflammation, and thus are not RA-specific. In contrast, Dominique Baeten (Ghent, Belgium) provided evidence that certain intracellular citrullinated proteins are generated only in RA. The precise nature of these RA-specific proteins, however, is still unclear.

Citrullinated proteins are also generated in arthritic animals and the citrullination was shown to increase arthritogenicity (Helena Erlandsson, Stockholm, Sweden). In this context it was interesting to hear the first preliminary results of studies with PAD knockout mice (Brian Werneburg, Ridgefield, USA) and PAD overexpression (Han Zendman, Nijmegen, The Netherlands).

\section{CITRULLINATION AND MULTIPLE SCLEROSIS}

The subject of citrullination and MS was introduced by Jon Laman (Rotterdam, The Netherlands) and George Harauz (Guelph, Canada). MS is a chronic inflammatory disorder of the CNS, characterised by destruction of the myelin sheath. The latter is formed by a cell layer that covers the axons of neurones. These cells, oligodendrocytes in the CNS, and Schwann cells in the peripheral nervous system, provide electrical insulation to the neurone, which is important for adequate conduction of nerve impulses. Demyelination causes loss of nerve signals, which in turn results in many different clinical manifestations (for example, loss of motor function, loss of vision, loss of sensation and paraesthesia).

The cells of the myelin sheath form a multibilayer membrane structure around the axons consisting of lipidprotein layers. One of the major protein components is $\mathrm{MBP}$, and this protein is known to undergo various posttranslational modifications (phosphorylation, deamidation, arginine methylation, acetylation at its $\mathrm{N}$-terminus, and also citrullination) (reviewed by Harauz et $a l^{2}$ ).

In healthy adult humans about $18 \%$ of the MBP molecules have six (out of 19) of their arginine residues converted to citrulline (MBP-cit 6 ). The remaining MBP molecules do not contain citrulline. In patients with MS the proportion of MBP-cit ${ }_{6}$ is increased to $45 \%$ of total MBP. In addition, in the rare Marburg type of acute fulminating MS, $80 \%$ of the MBP molecules are heavily citrullinated (MBP- $\left.\mathrm{cit}_{18}\right)$.

The decreased net positive charge of MBP-cit ${ }_{6}$ weakens its interactions with the negatively charged phospholipids. Complexes containing $\mathrm{MBP}-$ cit $_{6}$ are not as densely packed as those formed with non-citrullinated MBP. In addition, the partly unfolded structure of $\mathrm{MBP}^{-c_{1}}{ }_{6}$ renders it more susceptible to proteolytic degradation, which might directly be related to the observed demyelination of axons in the brain of patients with MS. Reinout Raijmakers (Nijmegen, The Netherlands) and Anthony Nicholas (Birmingham, USA) used experimental autoimmune encephalomyelitis (EAE) mouse models for studying the process of citrullination in MS. They presented data showing that in these animals not only MBP but also other CNS proteins, such as the astrocytespecific glial fibrillary acidic protein, are citrullinated. These data confirm the notion that citrullination of brain proteins is intimately associated with the demyelination process during the development of MS.

\section{CITRULLINATION AND RHEUMATOID ARTHRITIS}

Several speakers presented data on the antibodies to citrullinated proteins (ACPA) or cyclic citrullinated peptides (CCP) in patients with RA. After an overview of historical data (Guy Serre, Toulouse, France) and the clinical usefulness of these antibodies (Peter Schur, Boston, USA), additional new data were presented by a number of speakers. From these it can be concluded that anti-CCP antibodies and ACPA are extremely specific for RA ( $\sim 98 \%)$ and present very early in the disease (Dörte Hamann and Ben Dijkmans, Amsterdam, The Netherlands; Björn Svensson, Lund, 
Sweden; Floris van Gaalen, Leiden, The Netherlands). With more sophisticated and effective treatments becoming available and with the understanding that early intervention is crucial in preventing irreversible joint damage, early and accurate diagnosis is essential. In that respect the antibodies to citrullinated proteins/peptides can be very helpful in early diagnosis of RA when only a few clinical symptoms are manifest.

Valerie Nell (Vienna, Austria) presented data on the diagnostic and prognostic significance of ACPA. ACPA positive patients with RA appear to have a more erosive disease progression, and the detection of these antibodies can therefore be used in clinical practice to help in planning a therapeutic strategy. However, the level of these antibodies does not seem to decrease significantly during the treatment of patients (Filip de Keyser, Ghent, Belgium).

The question why antibodies to citrullinated antigens are so specific for RA was discussed by Lars Klareskog (Stockholm, Sweden), and René Toes (Leiden, The Netherlands). They showed that there is a functional link between RA-specific HLA haplotypes (in particular, HLADR4) and the production of ACPA. The combination of genetic status and positive ACPA in an individual subject may thus result in a much higher chance of developing RA.

Other genetic predispositions were also discussed. Most interestingly, immunisation of HLA-DR4 transgenic mice with citrullinated human fibrinogen induced mild arthritis, first detectable at day 70 after immunisation, which was associated with the development of pannus tissue and bone erosions detectable at day 150 (Jonathan Hill, London, Canada). It has been shown earlier that one haplotype of the citrullinating enzyme PAD4 was associated with susceptibility for RA in a Japanese population. ${ }^{4}$ In theory, this could result in more PAD4 enzyme being produced, and subsequently lead to increased citrullination of proteins and a higher chance of developing anti-CCP antibodies. Although the same haplotypes can also be found in a cohort of European patients, they were not associated with RA or with the erosive outcome in RA (Anne Barton, Manchester, UK). The session on autoantibodies to citrullinated proteins/ peptides ended with the first report on $\mathrm{T}$ cell epitopes on citrullinated fibrinogen in patients with RA (Jean Roudier, Marseille, France). He reported that (a) shared epitope positive HLA-DR alleles are associated with anti-citrullinated fibrinogen antibodies in patients with RA; (b) HLADRB ${ }^{*} 0404$, the most strongly associated allele, is also the one that binds the highest number of peptides from the fibrinogen A and B chains; and (c) the presence of citrulline residues is not critical for fibrinogen peptides to bind shared epitope containing HLA-DR alleles.

It has been shown in several studies that the autoantibodies to citrullinated proteins/peptides are locally produced. Similarly, it has been shown that citrullinated autoantigens such as fibrin and vimentin are present in the synovium as well. Certainly, the synovial "citrullinome" comprises many more proteins. Gerd Burmester (Berlin, Germany) lifted a tip of the veil by discussing the presence of citrullinated proteins in synovial vesicles, called exosomes. Along with citrullinated fibrin and fibrinogen fragments, a CD5-like antigen was also found in a citrullinated form. It is therefore very likely that immune complexes are formed in the RA synovium, although experimental evidence for this is not yet available. Future studies should investigate the presence of such immune complexes and their ability to stimulate the immune system in such a way that the inflammation perseveres. When this is proved it will be worthwhile investigating how the formation of such immune complexes can be blocked. This might lead to a new, specific treatment of RA.

\section{WHAT CAN WE EXPECT OF CITRULLINATION IN THE FUTURE?}

William Robinson (Stanford, USA) has applied the microarray technique to profile autoantibody responses in cohorts of patients with RA and control patients. In addition to demonstrating that autoantibody targeting of citrullinated autoantigens is sensitive and specific for RA, patients with RA with ACPA responses frequently exhibit autoantibody reactivity against multiple citrullinated peptides and/or proteins contained on the arrays. He also presented data using a bead array to profile serum cytokines in RA, and observed raised levels of proinflammatory and immunomodulatory cytokines in about one third of patients with RA. Raised levels of serum cytokines were associated with presence of anti-citrulline autoantibody responses together with clinical features predictive of a more severe disease course.

The potential role of citrullination and anti-citrulline immune responses in the pathogenesis of RA was discussed extensively throughout the meeting. The data showing that anti-CCP antibodies can pre-date clinical RA by years in certain patients suggests that anti-citrulline responses may have a pathogenic role. The data presented demonstrating antibody activity to citrulline substituted fibrinogen peptides also supports this view. Although a substantial body of suggestive data exists, experimental data demonstrating a clear pathogenic role in autoimmune arthritis in mice or humans has not been presented to date. Adenoviral delivery of PAD4 to mouse joints did not evoke arthritis, and the PAD2 and PAD4 deficient mice did not exhibit clear alterations in the severity of EAE. Many investigators in the field are designing and performing experiments attempting to answer this question.

\section{NEW THERAPEUTIC APPROACHES IN MS AND RA}

Paul Utz (Stanford, USA) presented an overview of proteomic technologies to study RA and other autoimmune diseases. These technologies included mass spectrometry, antibody arrays, antigen arrays, lysate arrays, phosphoprotein flow cytometry, and other technologies. Utz also presented in depth development of "reverse phase lysate" protein array technology to study signal transduction in autoimmune diseases. Reverse phase lysate arrays are produced by printing lysates, generated from $\mathrm{T}$ or other immune cells over a time course after stimulation, in ordered arrays on nitrocellulose coated microscope slides. These arrays are probed with multiple distinct phosphoprotein-specific antibodies to profile kinase, phosphatase, and other signalling pathway molecule activation states. Finally, he presented an application of the use of this technology to analyse rare primary cell populations as shown in a study of differential STAT protein phosphorylation in interleukin 2 stimulated CD4+/CD25+ regulatory $\mathrm{T}$ cells.

Although recombinant antibodies and soluble receptors enable specific blockade of cytokines and certain receptors, most of these treatments globally modulate immune function and are thereby not fundamental or curative. William Robinson (Stanford, USA) reviewed the development of antigen-specific tolerising DNA vaccines for the treatment of human autoimmune disease. ${ }^{5}$ Tolerising DNA vaccines deliver an autoantigen encoded in a mammalian expression vector by intramuscular injection. These vaccines have demonstrated efficacy in murine models of MS, autoimmune diabetes, and RA. Mouse model data demonstrate that such vaccines can induce specific anergy of the autoreactive T cells. They also reduce epitope spreading of autoreactive B cell responses. 


\section{SHOULD ANTI-CCP/ACPA POSITIVITY BECOME A CLASSIFICATION CRITERION FOR RA?}

The meeting ended with a comprehensive discussion, led by David Pisetsky (Durham, USA), Josef Smolen (Vienna, Austria), and Peter Lipsky (Bethesda, USA), on the question of whether anti-CCP/ACPA positivity should become a classification criterion for RA. There was general agreement that because the anti-CCP/ACPA antibody system is more specific than, and at least as equally sensitive as, the rheumatoid factor, it could and probably should replace rheumatoid factor or be added to the existing classification criteria. However, because so many other new tools of possible relevance for the classification and diagnosis of RA (for instance in the field of imaging and genetics) have become available recently, the majority favoured that such additions should be part of a comprehensive revision of the 1987 ACR criteria. The ACR and EULAR are already discussing this topic.

The organisers wish to thank the following sponsors: Abbott, AxisShield, EuroDiagnostics, Medical and Biological Labs Co Ltd, MSD, Nationaal Reumafonds, Organon, Pfizer, Pharmacia Diagnostica Roche, Schering Plough, Wyeth, Landelijk Katholiek Reumacentrum
E R Vossenaar,
Department of Biochemistry, Radboud University Nijmegen, Nijmegen

The Netherland's

W H Robinson,

Division of Immunolgy and Rheumatology, Stanford University School'of Medicine, Stanford, USA; Palo Alto Veterans Affairs Health Care System, Palo Alto, California, USA

\section{REFERENCES}

1 Nijenhuis S, Zendman AJW, Vossenaar ER, Pruiin GJM, van Venrooii WJ. Autoantibodies to citrullinated proteins in rheumatoid arthritis: clinical performance and biochemical aspects of an RA-specific marker. Clin Chim Acta 2004;350:17-34.

2 Harauz G, Ishiyama N, Hill CM, Bates IR, Libich DS, Fares C. Myelin basic protein-diverse conformational states of an intrinsically unstructured protein and its roles in myelin assembly and multiple sclerosis. Micron 2004;35:503-42.

3 Vossenaar ER, Zendman AJW, van Venrooii WJ, Pruiin GJM. PAD, a growing family of citrullinating enzymes. BioEssays 2003;25:1106-18.

4 Yamada R, Suzuki A, Chang X, Yamamoto K. Peptidylarginine deiminase type 4: identification of a rheumatoid arthritis-susceptible gene. Trends Mol Med 2003;9:503-8.

5 Robinson WH, Fontoura P, Lee BJ, de Vegvar HE, Tom J, Pedotti R, et al. Protein microarrays guide tolerizing DNA vaccine treatment of autoimmune encephalomyelitis. Nat Biotechnol 2003;21:1033-9.

\section{Autologous Stem cell Transplantation International Scleroderma (ASTIS) trial: hope on the horizon for patients with severe systemic sclerosis}

T he ASTIS (Autologous Stem cell Transplantation International Scleroderma) trial was launched in 2001 under the auspices of the European League Against Rheumatism (EULAR) and the European Group for Blood and Bone Marrow Transplantation (EBMT) to compare safety and efficacy of high dose immunosuppressive treatment (HDIT) and autologous haematopoietic stem cell transplantation (HSCT) with $12 \times$ monthly treatments with intravenous pulse cyclophosphamide $\left(750 \mathrm{mg} / \mathrm{m}^{2}\right)$. The trial builds upon the results from previous pilot studies conducted in various European centres, which demonstrated the feasibility and potential long term benefits of HDIT + HSCT in patients with severe rheumatic autoimmune diseases, including progressive systemic sclerosis. To combine safety and efficacy, a medium intensity regimen was chosen to achieve immunoablation in the ASTIS protocol, consisting of high dose cyclophosphamide $\left(2 \times 2 \mathrm{~g} / \mathrm{m}^{2}\right)+$ Filgrastim (granulocyte-colony stimulating factor; $10 \mu \mathrm{g} / \mathrm{kg}$ ) for mobilisation of haematopoietic stem cells, leukapheresis, and CD34 selection of the apheresis product, conditioning with high dose cyclophosphamide $(200 \mathrm{mg} / \mathrm{kg}$, rabbit ATG $7.5 \mathrm{mg} / \mathrm{kg}$ ), followed by HSCT.

The ASTIS trial targets patients with early diffuse systemic sclerosis at risk of early mortality. These include patients with disease duration of $\leqslant 4$ years, a modified Rodnan skin score (mRSS) of at least 15 (out of a maximum of 51), evidence of heart, lung, or kidney disease; and patients with a maximum disease duration of 2 years, an mRSS of 20 or more plus laboratory signs of acute phase reaction. Exclusion criteria are end stage organ failure, extensive pretreatment with cyclophosphamide (over $5 \mathrm{~g}$ intravenously or 3 months' oral treatment), and common reasons that preclude participation in a trial.

The primary end point is event-free survival, defined as the time in days since randomisation until death or the development of irreversible end stage organ failure. The major secondary end points are progression-free survival, treatment related mortality, and toxicity according to WHO criteria. It is postulated that HDIT + HSCT is a better treatment than pulse therapy because of its more profound perturbation of the immune system, although other mechanisms may also be involved.

Interim safety analyses are conducted after groups of 20 patients have been enrolled, and the results from the first analysis were presented at the 2004 EULAR meeting in Berlin. At the time of writing (July 2005), 55 patients have been randomised in 20 European centres, 25 to the investigational arm and 30 to the control arm. No treatment related mortality or unexpected toxicity has been seen in either treatment arm (median follow up 24 months, range 1-51).

EBMT registered transplant centres with expertise in the treatment of severe scleroderma are highly encouraged to participate in this unique joint effort, which will remain open until 2008. Information on the trial is regularly updated on the trial's website: http://www.astistrial.com (accessed 15 August 2005). It is hoped that the ASTIS trial, and its North American counterpart (the SCOT trial) will result in more effective treatment of patients with severe systemic sclerosis, with the aim of inducing a durable remission in the majority of patients treated.

\section{ACKNOWLEDGEMENTS}

The ASTIS trial is supported by grants from Amgen Europe (unrestricted educational grant), the Horton Foundation in Switzerland, the Groupe Francais de Recherche sur la Sclérodermie, and the Direction de la Recherche Clinique Assistance Publique Hopitaux de Paris in France, and EULAR.

J M van Laar,
Leiden University Medical Centre, Leiden, The Netherlands
D Farge,
Hopital St Lovis, Paris, France
A Tyndall,
Felix-Platter Spital, Basel, Switzerland
University Medical Centre, Albinusdreef 2, 2333 ZA Leiden,
The Netherlands; i.m.van_laar@lumc.nl

